\section{Heterocycles in materials chemistry}

Materials science is contemporarily understood as an interdisciplinary branch of the research related to methods and problems commonly elaborated in physics, biology, chemistry, metallurgy, and mineralogy; in addition, an important role is also attributed to engineering. In the recent two decades rapid development of materials science was observed, and from the view of chemistry, this statement is valid for both inorganic and organic compounds.

Whereas inorganic compounds form a base for the development of ceramics, magnetic materials, metallic alloys, and carbon materials, organic compounds determine trends in the developments related to the fields of biomaterials, semiconductors, molecular probes, liquid crystals, new rubbers, plastics, etc.

Heterocyclic chemistry is currently considered as one of the most important parts of organic chemistry. Its origin relates mainly to organic synthesis, chemistry of natural products, medicinal chemistry, and polymer chemistry. The discovery of furfural and determination of its structure by Döbereiner (Döbereiner, J. W. Ber. Dtsch. Chem. Ges. 1832, 3, 141) was published only a few years after the milestone finding by F. Wöhler that inorganic ammonium cyanate can be converted into organic urea by heating in a glassware, outside of a living organism.

Currently, heterocyclic chemistry offers powerful tools, which are efficiently applied for the preparation of new materials with defined physicochemical properties, and this section of chemistry is of great importance for the civilization development and growing living standard of societies worldwide.

In general, diversely functionalized derivatives of aromatic sulfur and nitrogen heterocycles (e.g. thiophenes or selenothiophenes, and carbazoles, pyrroles or pyridines,

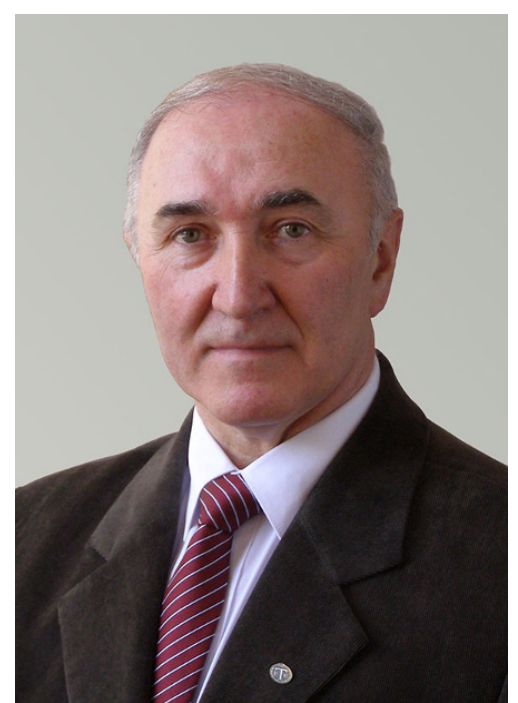

respectively) are recognized as heterocyclic compounds with key importance for the rapid development of new technologies, which are widely applied in materials chemistry.

The collection of review articles and original publications placed in this "Thematic issue" presents a useful overview on current problems related to new developments in the research focused on materials chemistry, performed in laboratories of several groups working in China, Czechia, France, Germany, Poland, and Russia. On behalf of the Editorial Board as well as Editorial Office, I express my sincere thanks to all authors for the acceptance of my invitation to contribute to this issue and for an efficient collaboration in the course of its preparation in the final form. The presented publications demonstrate once more that chemistry of heterocyclic compounds offers attractive methods for new solutions in the field of materials chemistry and allow to hope that they will be further developed by the next generation of chemists, starting right now professional adventures with the synthesis of heterocyclic compounds and studies on their physicochemical properties. Good luck for new achievements and successful developments of materials oriented heterocyclic chemistry!

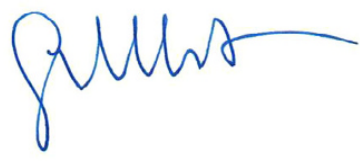

Editor of the thematic issue Professor, Dr. Hab.

Grzegorz Mlostoń Faculty of Chemistry University of Lódź, Poland 